\title{
Fluctuations in High Energy Nucleus-Nucleus Collisions from Microscopic Transport Approaches
}

\author{
Volodymyr Konchakovski* \\ Bogolyubov Institute for Theoretical Physics, Kiev, Ukraine \\ E-mail: voka@univ.kiev.ua
}

\begin{abstract}
Event-by-event multiplicity fluctuations in nucleus-nucleus collisions are studied within the HSD and UrQMD transport models. The scaled variances of negative, positive, and all charged hadrons in $\mathrm{Pb}+\mathrm{Pb}$ at $158 \mathrm{AGeV}$ are analyzed in comparison to the data from the NA49 Collaboration. We find a dominant role of the fluctuations in the nucleon participant number for the final hadron multiplicity fluctuations. This fact can be used to check different scenarios of nucleus-nucleus collisions by measuring the final multiplicity fluctuations as a function of collision centrality. The analysis reveals surprising effects in the recent NA49 data which indicate a rather strong mixing of the projectile and target hadron production sources even in peripheral collisions.
\end{abstract}

Correlations and Fluctuations in Relativistic Nuclear Collisions

July 7-9 2006

Galileo Galilei Institute, Florence, Italy

\footnotetext{
* Speaker.
} 


\section{Introduction}

Here I present the results [1] on particle number fluctuations in high energy nucleus-nucleus $(\mathrm{A}+\mathrm{A})$ collisions within the HSD [2] and UrQMD [3] transport approaches. The analysis of fluctuations is an important tool to study a physical system created in high energy nuclear collisions. Recently, preliminary NA49 data on particle number fluctuations in $\mathrm{Pb}+\mathrm{Pb}$ collisions at $158 \mathrm{~A} \mathrm{GeV}$ for different centralities have been presented [4] which are in surprising disagreement with the results of both microscopic transport models that have been shown to reproduce both the different particle multiplicities and longitudinal differential rapidity distributions for central collisions of $\mathrm{Au}+\mathrm{Au}($ or $\mathrm{Pb}+\mathrm{Pb})$ collisions from AGS to SPS energies rather well [5].

The fluctuations in high energy particle and nuclear collisions (see, e.g., Refs. [6, 7, 8, 9 , 10, 11, 12, 13, 14, 15] and references therein) are studied on an event-by-event basis: a given observable is measured in each event and the fluctuations are evaluated for a specially selected set of these events. The statistical model has been successfully used to describe the data on hadron multiplicities in relativistic A+A collisions (see, e.g., Ref. [16] and a recent review [17]) as well as in elementary particle collisions [18]. This gives rise to the question whether the fluctuations, in particular the multiplicity fluctuations, do also follow the statistical hadron-resonance gas results [19]. The statistical fluctuations can be closely related to phase transitions in QCD matter, with specific signatures for 1-st and 2-nd order phase transitions as well as for the critical point [9, 10].

In addition to the statistical fluctuations, the complicated dynamics of $\mathrm{A}+\mathrm{A}$ collisions generates dynamical fluctuations. The fluctuations in the initial energy deposited inelastically in the statistical system yield dynamical fluctuations of all macroscopic parameters, like the total entropy or strangeness content. The observable consequences of the initial energy density fluctuations are sensitive to the equation of state of the matter, and can therefore be useful as signals for phase transitions [15]. Even when the data are obtained with a centrality trigger, the number of nucleons participating in inelastic collisions still fluctuates considerably. In the language of statistical mechanics, these fluctuations in participant nucleon number correspond to volume fluctuations. Secondary particle multiplicities scale linearly with the volume, hence, volume fluctuations translate directly to particle number fluctuations.

Our aim is to study the particle number fluctuations in $\mathrm{Pb}+\mathrm{Pb}$ collisions at $158 \mathrm{AGeV}$ within both the HSD and UrQMD transport models. We check the robustness of the two approaches and derive physical consequences from the results of the HSD and UrQMD simulations. Then we formulate a general picture of particle number fluctuations in different scenarios for $\mathrm{A}+\mathrm{A}$ collision processes.

\section{HSD and UrQMD results in comparison to the NA49 Data}

In each $\mathrm{A}+\mathrm{A}$ event only a fraction of all $2 A$ nucleons (the participant nucleons) interact. We denote the number of participant nucleons from the projectile and target nuclei as $N_{P}^{\text {proj }}$ and $N_{P}^{\text {targ }}$, respectively. Those nucleons which do not interact are called spectator nucleons. Their numbers are related to the participant numbers as $N_{S}^{p r o j}=A-N_{P}^{p r o j}$ and $N_{S}^{\text {targ }}=A-N_{P}^{\text {targ }}$. The trivial geometrical fluctuations due to impact parameter variations usually dominate in high energy $\mathrm{A}+\mathrm{A}$ collisions and mask the fluctuations of interest. One cannot fix the impact parameter experimentally, but 
even for a fixed impact parameter the number of participants must fluctuate from event to event. Moreover, the numbers of the projectile and the target participants differ in a given event. This is caused by fluctuations in the initial states of the colliding nuclei and the probabilistic character of the various hadron-hadron collision processes.

The NA49 Collaboration has tried to minimize the event by event fluctuations of the number of nucleon participants in measuring the multiplicity fluctuations. Samples of collisions with a fixed number of projectile spectators, $N_{S}^{p r o j}=$ const , and thus a fixed number of projectile participants, $N_{P}^{p r o j}=A-N_{S}^{p r o j}$, were selected. This selection is possible in fixed target experiments, where $N_{S}^{p r o j}$ is measured by a Zero Degree Veto Calorimeter, which covers the projectile fragmentation domain.
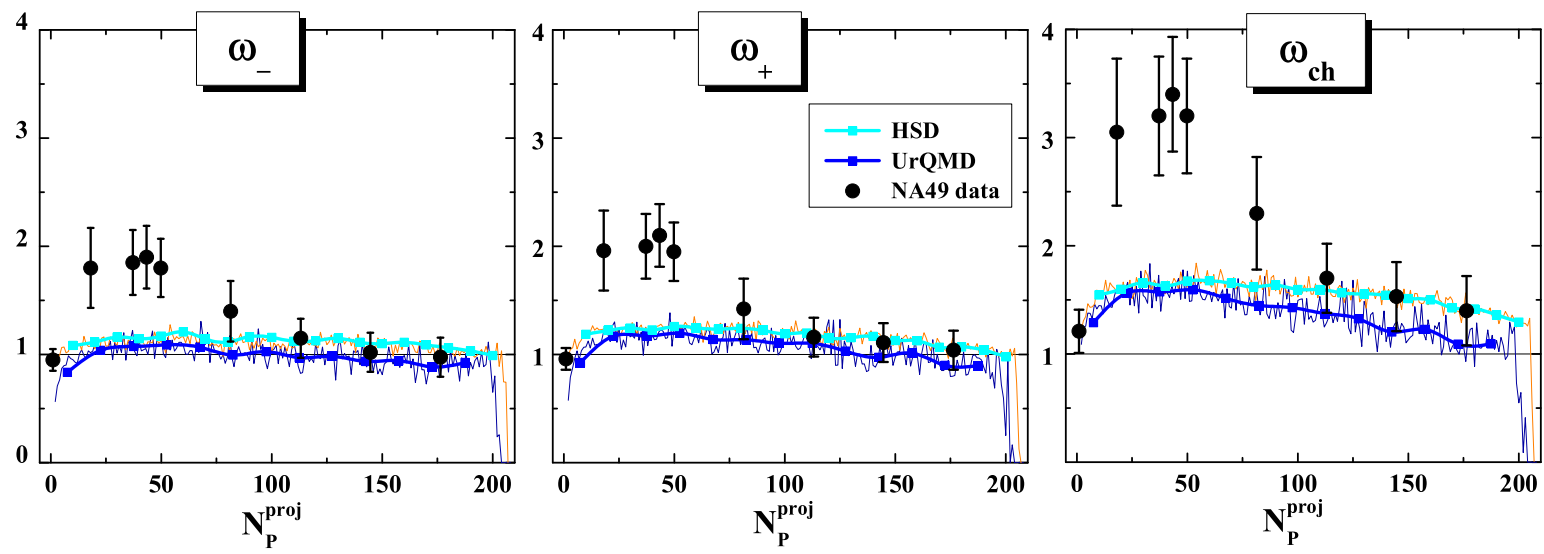

Figure 1: The results of the HSD and UrQMD simulations are shown for $\omega_{-}, \omega_{+}$, and $\omega_{c h}$ in $\mathrm{Pb}+\mathrm{Pb}$ collisions at $158 \mathrm{AGeV}$ as functions of $N_{P}^{p r o j}$. The black points are the NA49 data.

From an output of the HSD and UrQMD minimum bias simulations we form the samples of $\mathrm{Pb}+\mathrm{Pb}$ events with fixed values of $N_{P}^{\text {proj }}$. In Fig. 1 we present the HSD and UrQMD results and compare them with the NA49 data for the scaled variances of negatively, positively, and all charged particles in $\mathrm{Pb}+\mathrm{Pb}$ collisions at $158 \mathrm{AGeV}$. The average values:

$$
\left\langle N_{i}\right\rangle,(i=+,-, c h),
$$

where brackets denote the averaging in the model simulations; and variances:

$$
\operatorname{Var}\left(N_{i}\right) \equiv\left\langle N_{i}^{2}\right\rangle-\left\langle N_{i}\right\rangle^{2}
$$

are calculated for the samples of collision events with fixed values of the projectile participants, $N_{P}^{p r o j}$, and scaled variances are by definition,

$$
\omega_{i} \equiv \operatorname{Var}\left(N_{i}\right) /\left\langle N_{i}\right\rangle
$$

Note that $\omega=1$ for the Poisson multiplicity distribution, $P(N)=\exp (-\bar{N}) \bar{N}^{N} / N$ ! . 
The final particles in the HSD and UrQMD simulations are accepted at rapidities $1.1<y<2.6$ (we use particle rapidities in the $\mathrm{Pb}+\mathrm{Pb}$ c.m.s. frame) in accord to the NA49 transverse momentum filter [4]. This is done to compare the HSD and UrQMD results with the NA49 data. The HSD and UrQMD simulations both show flat $\omega_{i}$ values, $\omega_{-} \approx \omega_{+} \approx 1.2, \omega_{c h} \approx 1.5$, and exhibit almost no dependence on $N_{P}^{p r o j}$. The NA49 data, in contrast, exhibit an enhancement in $\omega_{i}$ for $N_{P}^{\text {proj }} \approx 50$. The data show maximum values, $\omega_{-} \approx \omega_{+} \approx 2$ and $\omega_{c h} \approx 3$, and a rather strong dependence on $N_{P}^{p r o j}$.
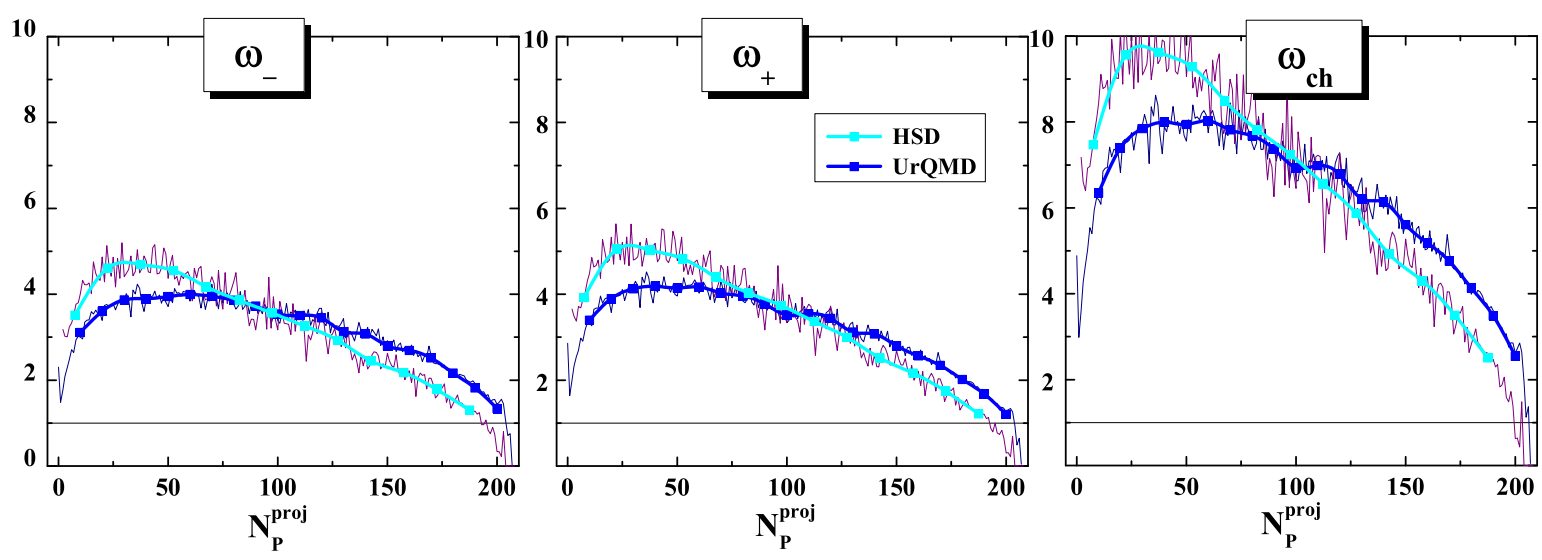

Figure 2: The results of the HSD and UrQMD simulations are shown for $\omega_{-}, \omega_{+}$, and $\omega_{c h}$ in $\mathrm{Pb}+\mathrm{Pb}$ collisions at $158 \mathrm{AGeV}$ as functions of $N_{P}^{p r o j}$ in full $4 \pi$ acceptance.

Fig. 2 shows results of the HSD and UrQMD simulations for the full $4 \pi$ acceptance for final particles. We see even larger fluctuations follow from the HSD and UrQMD simulations for the full acceptance of final particles then from the experiment.

\section{Multiplicity fluctuations in projectile and target hemispheres}

Let us consider now the fluctuations of the particle multiplicities in the projectile $(y>0)$ and target $(y<0)$ hemispheres. Both simulations, HSD and UrQMD, give very different results for the particle number fluctuations in the projectile and target hemispheres. The particle number fluctuations in the target hemispheres are much stronger (see Fig. 3) than those in the projectile hemispheres. There is also a strong $N_{P}^{p r o j}$-dependence of $\omega_{i}$ in the target hemisphere, which is almost absent for the $\omega_{i}$ in the projectile hemisphere.

Fig. 4 presents the scaled variances $\omega_{P}^{\text {targ }}$ calculated within the HSD and UrQMD models as functions of $N_{P}^{p r o j}$. The fluctuations of $N_{P}^{\text {targ }}$ are quite strong; the largest value of $\omega_{P}^{\text {targ }}=3-3.5$ occurs at $N_{P}^{\text {proj }}=20-30$.

As one can see from Fig. 4, in samples with $N_{P}^{p r o j}=$ const the number of target participants, $N_{P}^{\text {targ }}$, fluctuates considerably. Of course, this event selection procedure introduces an asymmetry between projectile and target participants: $N_{P}^{p r o j}$ is constant, whereas $N_{P}^{\text {targ }}$ fluctuates. And we see 

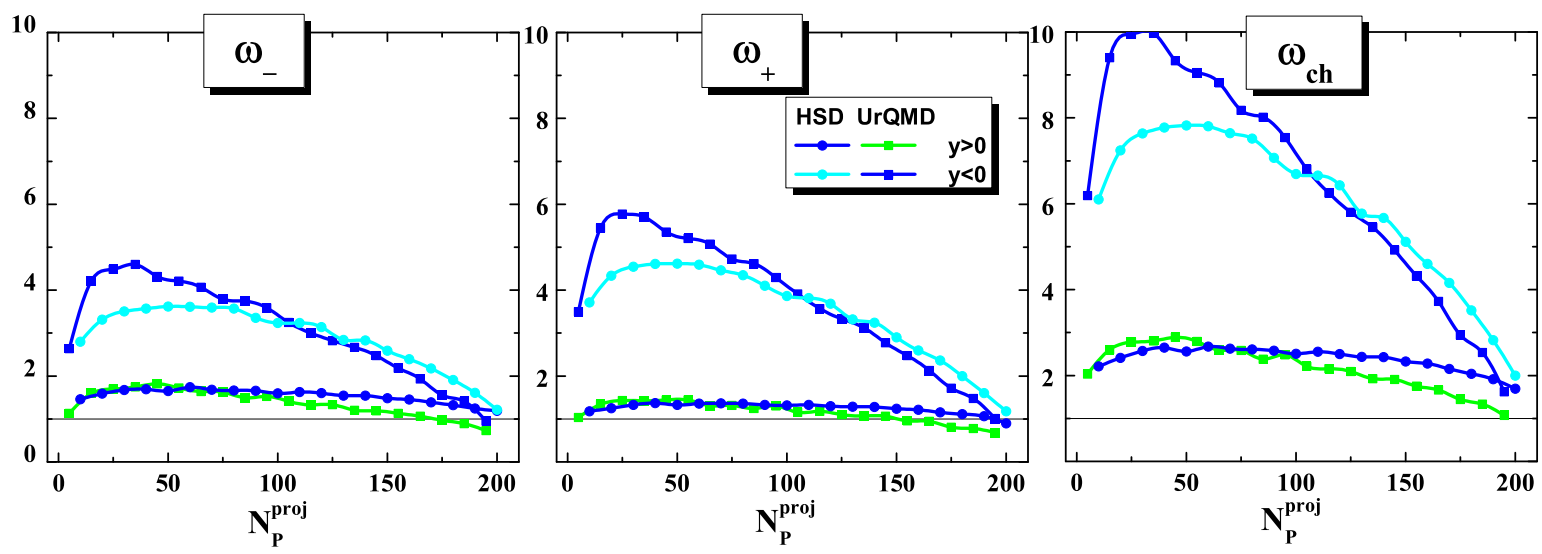

Figure 3: The scaled variances $\omega_{i}$ for the projectile (lower lines) and target (upper lines) hemispheres in the HSD and UrQMD simulations.

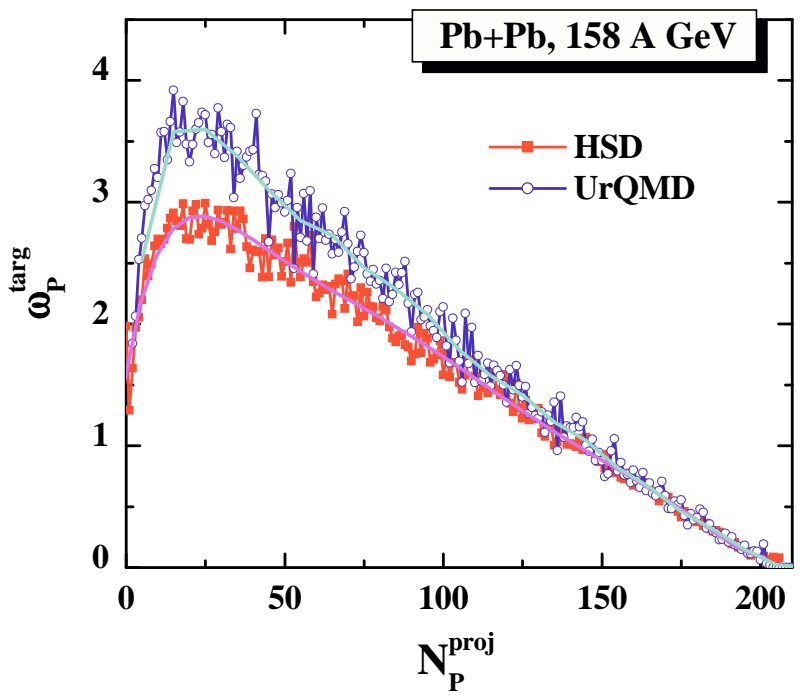

Figure 4: Scaled variance $\omega_{P}^{\text {targ }}$ for the fluctuations of the number of target participants, $N_{P}^{\text {targ }}$. HSD and UrQMD simulations show similar behavior of $\omega_{P}^{\text {targ }}$ as a function of $N_{P}^{\text {proj }}$.

the difference between the projectile and target hemispheres due to the asymmetry between projectile and target participants. The target participants, $N_{P}^{\text {targ }}$, play a quite small role for the particle production in the projectile hemisphere. Thus, the fluctuations of $N_{P}^{\text {targ }}$ have a small influence on the final multiplicity fluctuations in the projectile hemisphere, but they contribute very strongly to those in the target hemisphere.

To understand the nature of large fluctuations in the full acceptance within transport models let us consider of the independent source model. After simple operations [1] we get that scaled 
variance in full acceptance can be presented as:

$$
\omega_{i} \equiv \frac{\operatorname{Var}\left(N_{i}\right)}{\left\langle\overline{N_{i}}\right\rangle}=\omega_{i}^{*}+\omega_{P} n_{i},
$$

where $n_{i}$ - is the average number of particles of $i$-th type per participant. The total number of participants fluctuates due to the fluctuations of $N_{P}^{\text {targ }}$ (the values of $N_{P}^{p r o j}$ are fixed experimentally, as well as in the HSD and UrQMD simulations). One calculates the average values, $\left\langle N_{P}^{\text {targ }}\right\rangle \simeq N_{P}^{\text {proj }}$, and scaled variances, $\omega_{P}^{\text {targ }}$, for the target participants in both the HSD and UrQMD models (see Fig. 4).

The scaled variance $\omega_{P}$ for the total number of participants is easily found, $\omega_{P}=\omega_{P}^{\text {targ }} / 2$, as only a half of the total number, $N_{P}$, of participants, i.e., $N_{P}^{\text {targ }}$, does fluctuate.

Putting everything together we get:

$$
\omega_{i}=\omega_{i}^{*}+\frac{1}{2} \omega_{P}^{\text {targ }} n_{i}
$$

The average number of particles $n_{i}$ can be easily found from model calculations ([1]).

In peripheral $\mathrm{A}+\mathrm{A}$ collisions there are only few $\mathrm{N}+\mathrm{N}$ collisions, and rescatterings are rare, so that the picture of independent $\mathrm{N}+\mathrm{N}$ collisions looks reasonable. In this case, a hadron production source can be associated with a $\mathrm{N}+\mathrm{N}$ collision. The HSD results for $\omega_{i}^{*}$ per $\mathrm{N}+\mathrm{N}$ collision at 158 $\mathrm{GeV}$ :

$$
\omega_{c h}^{*}=2.5, \quad \omega_{-}^{*}=1.5, \quad \omega_{+}^{*}=1.1
$$

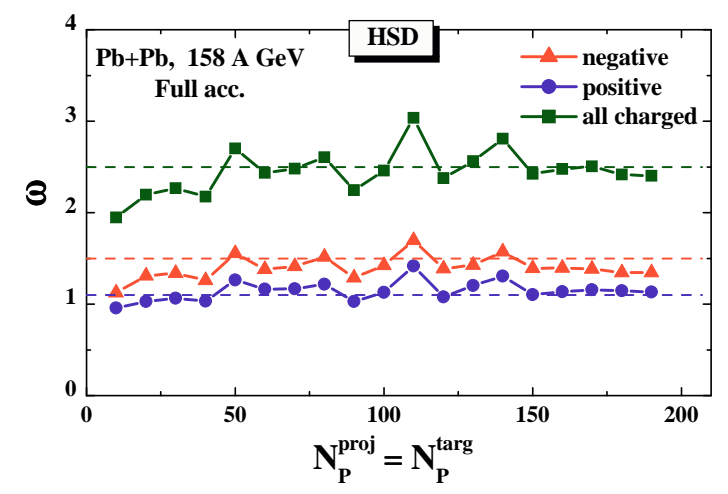

Figure 5: The circles, triangles, and boxes are the results of the HSD simulations for $\omega_{i}$ in full $4 \pi$ acceptance with $N_{P}^{\text {targ }}=N_{P}^{\text {proj }}$. The dashed lines correspond to $\omega_{i}^{*}$ taken from Eq. (3.3).

HSD events with fixed target participant number, $N_{P}^{\text {targ }}=N_{P}^{\text {proj }}$, exhibit much smaller multiplicity fluctuations (see 5). This is due to the fact that terms proportional to $\omega_{P}^{\text {targ }}$ in Eq. (3.2) do not contribute, and $\omega_{i}$ become approximately equal to $\omega_{i}^{*}$.

\section{Transparency, mixing and reflection in $\mathrm{A}+\mathrm{A}$}

Different models of hadron production in relativistic $\mathrm{A}+\mathrm{A}$ collisions can be divided into three limiting groups: transparency, mixing, and reflection models (see Ref. [20]). The first group assumes that the final longitudinal flows of the hadron production sources related to projectile and 
target participants follow in the directions of the projectile and target, respectively. We call this group of models transparency (T-)models. If the projectile and target flows of hadron production sources are mixed, we call these models the mixing (M-)models. Finally, one may even speculate that the initial flows are reflected in the collision process. The projectile related matter then flows in the direction of the target and the target related matter flows in the direction of the projectile. This class of models we call the reflection (R-)models. The rapidity distributions resulting from the T-, M-, and R-models are sketched in Fig. 6 taken from Ref. [20].

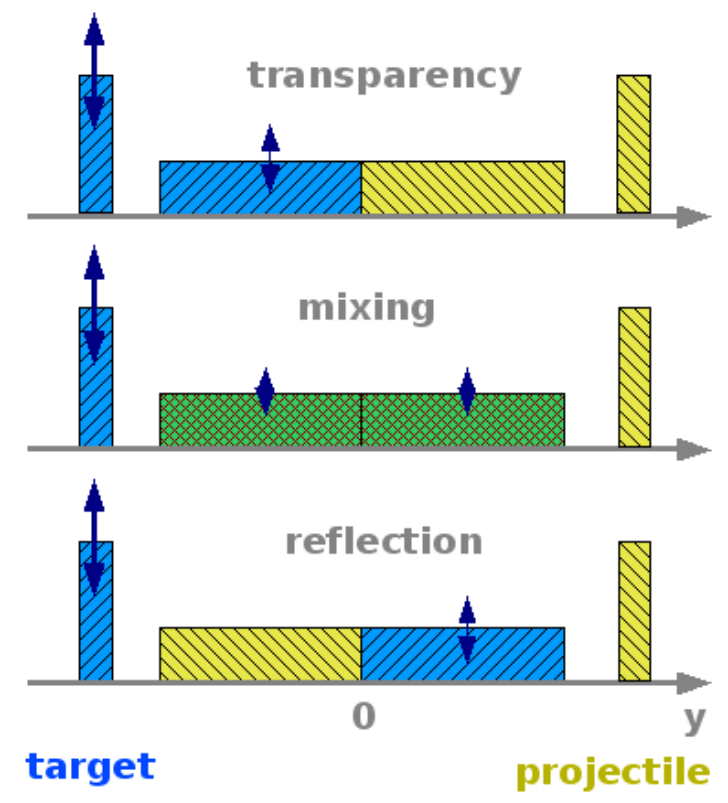

Figure 6: The rapidity distributions of the particle production sources in nucleus-nucleus collisions resulting from transparent, mixing, and reflection models (see Ref. [20] and text for details).

An asymmetry between the projectile and target participants introduced by the experimental selection procedure can be used to distinguish between projectile related and target related final state flows of hadron production sources as suggested in Ref. [20]. One expects large fluctuations of hadron multiplicities in the domain of the target related flow and small fluctuations in the domain of the projectile related flow. When both flows are mixed, intermediate fluctuations are predicted. The different scenarios are presented in Fig. 6. The multiplicity fluctuations measured in the projectile momentum hemisphere clearly are larger than those measured in the target hemisphere in T-models. The opposite relation is predicted for R-models, whereas for M-models the fluctuations in the projectile and target hemispheres are expected to be the same.

\section{Summary and conclusions}

The event-by-event multiplicity fluctuations in $\mathrm{Pb}+\mathrm{Pb}$ collisions at $158 \mathrm{AGeV}$ have been studied within the HSD and UrQMD transport models. The scaled variances of negative, positive, and all charged hadrons are analyzed in minimum bias simulations for samples of events with fixed 
numbers of the projectile participants, $N_{P}^{p r o j}$. This strong centrality trigger corresponds to the trigger of the NA49 Collaboration.

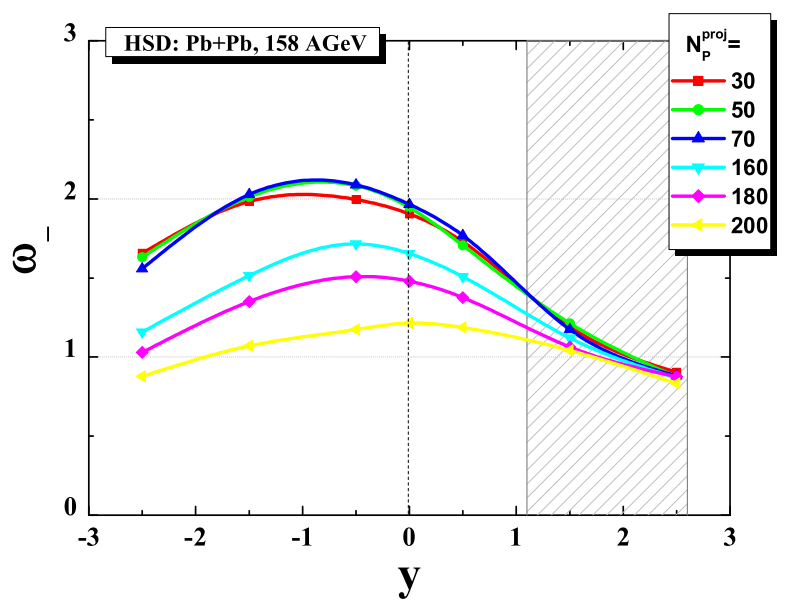

Figure 7: The results of the HSD and UrQMD simulations are shown for $\omega_{-}, \omega_{+}$, and $\omega_{c h}$ in $\mathrm{Pb}+\mathrm{Pb}$ collisions at $158 \mathrm{AGeV}$ as functions of $N_{P}^{\text {proj }}$ in full $4 \pi$ acceptance.

The samples with $N_{P}^{\text {proj }}=20-60$ show the large fluctuations of the number of target nucleons, $N_{P}^{\text {targ }}$, which participate in inelastic collisions, $\omega_{P}^{\text {targ }} \geq 2$. The final hadron multiplicity fluctuations exhibit analogous behavior, which explains the large values of the HSD and UrQMD scaled variances $\omega_{i}$ in the target hemispheres and in the full $4 \pi$ acceptance. On the other hand, the asymmetry between the projectile and target participants - introduced in the data samples by the trigger condition of fixed $N_{P}^{\text {targ }}$ - can be used to explore different dynamics of nucleus-nucleus collisions by measuring the final multiplicity fluctuations as a function of rapidity (cf. Fig. 7). This analysis reveals that the recent NA49 data indicate a rather strong mixing of the longitudinal flows of the projectile and target hadron production sources. This is so not only for central collisions in line with the HSD and UrQMD approaches [5] - but also for rather peripheral reactions. This sheds new light on the nucleus-nucleus reaction dynamics at top SPS energies for peripheral and mid-peripheral $\mathrm{Pb}+\mathrm{Pb}$ collisions. It demonstrates a significantly larger amount of mixing than is generated in simple hadron/string transport approaches.

The fluctuations of baryonic number and electric charge in high energy nucleus-nucleus collisions are also studied and are presented in [21].

\section{Acknowledgments}

I would like to thank V.V. Begun, M. Bleicher, E.L. Bratkovskaya, M. Gaździcki, M.I. Gorenstein, S. Haussler, B. Lungwitz and H. Stöker for fruitful collaboration. 


\section{References}

[1] V. P. Konchakovski, S. Haussler, M. I. Gorenstein, E. L. Bratkovskaya, M. Bleicher and H. Stoecker, Phys. Rev. C 73 (2006) 034902

[2] W. Ehehalt and W. Cassing, Nucl. Phys. A 602, 449 (1996); W. Cassing and E.L. Bratkovskaya, Phys. Rep. 308, 65 (1999).

[3] S.A. Bass et al., Prog. Part. Nucl. Phys.41, 255 (1998); M. Bleicher et al., J. Phys. G 25, 1859 (1999).

[4] M. Rybczynski et al. [NA49 Collaboration], J. Phys. Conf. Ser. 5, 74 (2005).

[5] H. Weber, E. L. Bratkovskaya, W. Cassing, and H. Stöcker, Phys. Rev. C67, 014904 (2003); E. L. Bratkovskaya, M. Bleicher, M. Reiter, S. Soff, H. Stöcker, M. van Leeuwen, S. A. Bass, and W. Cassing, Phys. Rev. C69, 054907 (2004); E. L. Bratkovskaya, M. Bleicher, W. Cassing, M. Reiter, S. Soff, H. Stöcker, Prog. Part. Nucl. Phys. 53, 225 (2004).

[6] M. Gaździcki and St. Mrówczyński, Z. Phys. C 26, 127 (1992);

[7] L. Stodolsky, Phys. Rev. Lett. 75, 1044 (1995); E.V. Shuryak, Phys. Lett. B 423, 9 (1998); St. Mrówczyński, Phys. Lett. B 430, 9 (1998).

[8] G. Baym and H. Heiselberg, Phys. Lett. B 469, 7 (1999).

[9] I.N. Mishustin, Phys. Rev. Lett. 82, 4779 (1999); Nucl. Phys. A 681, 56c (2001); H. Heiselberg and A.D. Jackson, Phys. Rev. C 63, 064904 (2001).

[10] M.A. Stephanov, K. Rajagopal, and E.V. Shuryak, Phys. Rev. Lett. 81, 4816 (1998); Phys. Rev. D 60, 114028 (1999); M.A. Stephanov, Acta Phys. Polon. B 35, 2939 (2004).

[11] S. Jeon and V. Koch, Phys. Rev. Lett. 83, 5435 (1999); ibid. 85, 2076 (2000).

[12] H. Heiselberg, Phys. Rep. 351, 161 (2001).

[13] S. Jeon and V. Koch, hep-ph/0304012, Review for Quark-Gluon Plasma 3, eds. R.C. Hwa and X.-N. Wang, World Scientific, Singapore.

[14] M. Bleicher et al., Nucl. Phys. A 638, 391 (1998); Phys. Lett. B 435, 9 (1998); Phys. Rev. C 62, 061902 (2000); ibid. 62, 041901 (2000); S. Jeon, L. Shi, and M. Bleicher, nucl-th/0506025; S. Haussler, H. Stoecker, and M. Bleicher, hep-ph/0507189.

[15] M. Gaździcki, M.I. Gorenstein, and St. Mrówczyński, Phys. Lett. B 585, 115 (2004); M.I. Gorenstein, M. Gaździcki, and O.S. Zozulya, ibid. 585, 237 (2004); M. Gaździcki, nucl-ex/0507017.

[16] H. Stöcker and W. Greiner, Phys. Rep. 137, 277 (1986); J. Cleymans and H. Satz, Z. Phys. C 57, 135 (1993); J. Sollfrank, M. Gaździcki, U. Heinz, and J. Rafelski, ibid. 61, 659 (1994); G.D. Yen, M.I. Gorenstein, W. Greiner, and S.N. Yang, Phys. Rev. C 56, 2210 (1997); F. Becattini, M. Gaździcki, and J. Solfrank, Eur. Phys. J. C 5, 143 (1998); G.D. Yen and M.I. Gorenstein, Phys. Rev. C 59, 2788 (1999); P. Braun-Munzinger, I. Heppe and J. Stachel, Phys. Lett. B 465, 15 (1999); P. Braun-Munzinger, D. Magestro, K. Redlich, and J. Stachel, ibid. 518, 41(2001); F. Becattini, M. Gaździcki, A. Keränen, J. Mannienen, and R. Stock, Phys. Rev. C 69, 024905 (2004).

[17] P. Braun-Munzinger, K. Redlich, J. Stachel, nucl-th/0304013, Review for Quark Gluon Plasma 3, eds. R.C. Hwa and X.-N. Wang, World Scientific,Singapore.

[18] F. Becattini, Z. Phys. C 69, 485 (1996); F. Becattini, U. Heinz, ibid. 76, 269 (1997); F. Becattini, G. Passaleva, Eur. Phys. J. C 23, 551 (2002). 
[19] V.V. Begun, M. Gaździcki, M.I. Gorenstein, and O.S. Zozulya, Phys. Rev. C 70, 034901 (2004); V.V. Begun, M.I. Gorenstein, and O.S. Zozulya, Phys. Rev. C 72, 014902 (2005); A. Keränen, F. Becattini, V.V. Begun, M.I. Gorenstein, and O.S. Zozulya, J. Phys. G 31, S1095 (2005); F. Becattini, A. Keränen, L. Ferroni, and T. Gabbriellini, Phys. Rev. C 72, 064904 (2005); V.V. Begun, M.I. Gorenstein, A.P. Kostyuk, and O.S. Zozulya, Phys. Rev. C 71, 054904 (2005); J. Cleymans, K. Redlich, and L. Turko, Phys. Rev. C 71, 047902 (2005); J. Phys. G 31, 1421 (2005); V.V. Begun, M.I. Gorenstein, A.P. Kostyuk, and O.S. Zozulya, J. Phys. G 32, 935 (2006). V.V. Begun and M.I. Gorenstein, Phys. Rev. C 73, 054904 (2006). V.V. Begun, M.I. Gorenstein, M. Hauer, V.P. Konchakovski, and O.S. Zozulya, Phys. Rev. C 74, 044903 (2006).

[20] M. Gaździcki and M.I. Gorenstein, Phys. Lett. B 640, 155 (2006), arXiv:hep-ph/0511058.

[21] V.P. Konchakovski, M.I. Gorenstein, E.L. Bratkovskaya, H. Stöcker, nucl-th/0606047. 